\title{
Bounds on Codes Based on Graph Theory
}

\author{
Salim Y. El Rouayheb \\ ECE Department \\ Texas A\&M University \\ College Station, TX 77843 \\ salim@ece.tamu.edu
}

\author{
Costas N. Georghiades \\ ECE Department \\ Texas A\&M University \\ College Station, TX 77843 \\ georghiades@ece.tamu.edu
}

\author{
Emina Soljanin \\ Math. Sc. Center \\ Bell Labs, Lucent \\ Murray Hill, NJ 07974 \\ emina@lucent.com
}

\author{
Alex Sprintson \\ ECE Department \\ Texas A\&M University \\ College Station, TX 77843 \\ spalex@ece.tamu.edu
}

\begin{abstract}
Let $A_{q}(n, d)$ be the maximum order (maximum number of codewords) of a $q$-ary code of length $n$ and Hamming distance at least $d$. And let $A(n, d, w)$ that of a binary code of constant weight $w$. Building on results from algebraic graph theory and Erdôs-ko-Rado like theorems in extremal combinatorics, we show how several known bounds on $A_{q}(n, d)$ and $A(n, d, w)$ can be easily obtained in a single framework. For instance, both the Hamming and Singleton bounds can derived as an application of a property relating the clique number and the independence number of vertex transitive graphs. Using the same techniques, we also derive some new bounds and present some additional applications.
\end{abstract}

\section{INTRODUCTION}

Let $\Sigma=\{0,1, \ldots, q-1\}$ be an alphabet of order $q$. A $q$-ary code $C$ of length $n$ and order $|C|$ is a subset of $\Sigma^{n}$ containing $|C|$ elements (codewords). The weight $w t(c)$ of a codeword $c$ is the number of its non-zero entries. A $w$ constant weight code is a code where all the codewords have the same weight $w$. The Hamming distance $d\left(c, c^{\prime}\right)$ between two codewords $c$ and $c^{\prime}$ is the number of positions where they have different entries. The minimum Hamming distance of a code $C$ is the largest integer $\Delta$ such that $\forall c, c^{\prime} \in C, d\left(c, c^{\prime}\right) \geq \Delta$.

Let $A_{q}(n, d)$ be the maximal number of codewords that a $q$-ary code of length $n$ and minimum Hamming distance $d$ can possibly contain ([1, Chapter 17]). $A(n, d, w)$ is defined similarly for binary codes with constant weight $w$. Finding the values of $A_{q}(n, d)$ and $A(n, d, w)$ is a basic problem in "classical" coding theory [2], [1].

Finding a general exact expression for the maximal order of codes is a difficult task. In fact, it was described in [4], as "a hopeless task". For this reason, much of the research done has focused on bounding these quantities.

The dual problem, consisting of finding the maximal order of a set of codewords satisfying an upper bound on their pairwise Hamming distance (anticodes), is well studied in extremal combinatorics. Surprisingly enough, it has a closed form solution [3], [4], [5].

Using tools from algebraic graph theory, we draw a link between the maximal order of codes and that of anti-codes. Then using results like the celebrated Erdős-ko-Rado theorem, we rederive some known inequalities on $A_{q}(n, d)$ and $A(n, d, w)$ and other similarly defined quantities and give some new bounds.

This paper is organized as follows. In Section [1] we briefly introduce some of the needed background in graph theory. In Section III we show how the tools introduced can be used to derive upper bounds on $A_{q}(n, d)$. In Sections [IV and $\mathrm{V}$ we derive bounds on the maximal size of constant and doubly constant weight codes, respectively. In Section VI, we show how the described techniques can be used to solve other problems. We conclude in Section VII, where we summarize our results and present some open questions.

\section{GRAPH THEORY BACKGROUND}

We start by giving a brief summary of some graph theoretical concepts and results that will be needed in this paper. For more details, we refer the interested reader to [6] and [7].

Let $G(V, E)$ be an undirected graph, where $V$ is its vertex set and $E$ is its edge set $(E \subseteq V \times V)$. We also use $\mathrm{V}(G)$ to denote the vertex set of $G$ and $\operatorname{E}(G)$ its edge set. If $\{u, v\}$ is an edge in $G$, i.e. $\{u, v\} \in E(G)$, we say that the vertices $u$ and $v$ are adjacent and write $u \sim v$.

The complement of a graph $G$ is the graph $\bar{G}$ defined over the same vertex set but where two vertices are adjacent in $\bar{G}$ iff they are not in $G$. We denote by $\omega(G)$ the clique number of a graph $G$, defined as the largest number of vertices of $G$ that are pairwise adjacent. In contrast $\alpha(G)$, the independence number of $G$, is the largest number of vertices in $G$ such that no two of them are adjacent. It can be easily seen that $\alpha(G)=\omega(\bar{G})$. In addition, the chromatic number $\chi(G)$ of $G$ is the minimum number of colors needed to color its vertices such that different colors are assigned to adjacent vertices.

Definition 1 (Graph Automorphism [7]): Let $G(V, E)$ be a graph and $\phi$ a bijection from $V$ to itself. $\phi$ is called an automorphism of $G$ iff

$$
\forall u, v \in V, u \sim v \Leftrightarrow \phi(u) \sim \phi(v) .
$$

The set of all automorphisms of $G$ is a group under composition; it is called the automorphism group of $G$ and it is denoted $\operatorname{Aut}(G)$. For example, the complete graph on $n$ vertices $K_{n}$ has $S_{n}$, the symmetric group of order $n$, as its automorphism group. In other words, $\operatorname{Aut}\left(K_{n}\right) \cong S_{n}$.

Definition 2 (Vertex Transitive Graph [7]): We say that graph $G(V, E)$ is vertex transitive iff

$$
\forall u, v \in V, \exists \phi \in \operatorname{Aut}(G) \text { s.t. } \phi(u)=v .
$$

Definition 3 (Cayley Graphs): Let $H$ be a group and $S \subset$ $H$ such that $S$ is closed under inversion and the identity element of $H 1_{H} \notin S$. The Cayley graph $\mathcal{C}(H, S)$ is the graph with vertex set $H$ and where for any $g, h \in H, g \sim h$ iff $h g^{-1} \in S$.

Next, we give without a proof an important result from [7] (Lemma 7.2.2) that will be instrumental in deriving our results.

Theorem 1: Let $G(V, E)$ be a vertex transitive graph, then

$$
\alpha(G) \omega(G) \leq|V(G)| \text {. }
$$




\section{BOUNDS ON CODES}

Definition 4 (Hamming Graph [2]): The Hamming graph $H_{q}(n, d), n \in \mathbb{N}$ and $1 \leq d \leq n$, has as vertices all the $q$-ary sequences of length $n$, and two vertices are adjacent iff their Hamming distance is larger or equal to $d$. That is, $V\left(H_{q}(n, d)\right)=\Sigma^{n}$, where $\Sigma=\{0,1, \ldots, q-1\}$. and $u \sim v$ iff $d(u, v) \geq d$.

Recall that $A_{q}(n, d)$ denotes the maximum number of codewords in a q-ary code of length $n$ and minimum Hamming distance $d$. When the subscript is omitted we assume $q=2$, i.e. $A(n, d)=A_{2}(n, d)$. It can be easily seen that $A_{q}(n, d)=$ $\omega\left(H_{q}(n, d)\right)$.

Let $S_{n, d}, 1 \leq d \leq n$, be a subset of the group $\left(\mathbb{Z}_{q}^{n},+\right)$, where addition is done modulo $q$, such that $S_{n, d}=\{s \in$ $\left.\mathbb{Z}_{q}^{n} ; w t(s) \geq d\right\}$. It is easy to check that $S_{n, d}$ is closed under inversion and does not contain the identity element (the all zero sequence). The next lemma asserts that the Hamming graph is in fact a Cayley graph.

Lemma 1: $H_{q}(n, d)=\mathcal{C}\left(\mathbb{Z}_{q}^{n}, S_{n, d}\right)$.

Proof: Take $\Sigma=\left(\mathbb{Z}_{q},+\right)$. The result then follows easily from the fact that $\forall x, y \in \mathbb{Z}_{q}^{n}, d(x, y)=w t(x-y)$.

Lemma 2: The Hamming graph $H_{q}(n, d)$ is vertex transitive.

Proof: Follows From Lemma 1 and the fact that Cayley Graphs are vertex transitive [7, Thm. 3.1.2].

For a clearer presentation, we also give here a direct proof. Take $\Sigma=\left(\mathbb{Z}_{q},+\right)$. And $\forall u, v, x \in \Sigma^{n}$, define the function $\phi_{u, v}(x)=x+v-u . \phi_{u, v}(x)$ is an automorphism of $H_{q}(n, d)$. In fact, $d\left(\phi_{u, v}(x), \phi_{u, v}(y)\right)=d(x+v-u, y+v-u)=$ $\mathrm{wt}(x+v-u-(y+v-u))=\mathrm{wt}(x-y)=d(x, y)$. Also, $\phi_{u, v}(x)$ takes $u$ to $v$.

Corollary 1: $A_{q}(n, d) \alpha\left(H_{q}(n, d)\right) \leq q^{n}$

Proof: Follows from Lemma 2 and Thm. 1

Notice that $\alpha\left(H_{q}(n, d)\right)$, the independence number of the Hamming graph $H_{q}(n, d)$, is actually the maximum number of sequences such that the Hamming distance between any two of them is at most $d-1$. Following [3], we define $N_{q}(n, s)$ to be the maximum number of $q$-ary sequences of length $n$ that intersect pairwise (have the same entries) in at least $s$ positions. It follows that

$$
\alpha\left(H_{q}(n, d)\right)=N_{q}(n, t) ; \quad \text { with } t=n-d+1
$$

Lemma 3 (Singleton Bound): $A_{q}(n, d) \leq q^{n-d+1}$

Proof: Consider the set $T(n, t)$ of $q$-ary sequences of length $n$ that all have the same element in the first $t=n-d+1$ entries. By definition, $N_{q}(n, t) \geq|T(n, t)|=q^{n-t}$. Then, by (1) and Corollary 1, $A_{q}(n, d) \leq \frac{q^{n}}{q^{n-t}}=q^{n-d+1}$.

Lemma 4 (Hamming Bound):

$$
A_{q}(n, d) \leq \frac{q^{n}}{\sum_{i=0}^{\left\lfloor\frac{d-1}{2}\right\rfloor}\left(\begin{array}{c}
n \\
i
\end{array}\right)(q-1)^{i}} .
$$

Proof: The proof is similar to that of Lemma 3 and is done by finding a different lower bound on $N_{q}(n, t)$. In fact, consider the ball $B(n, r)=\left\{x \in \Sigma^{n} ; w t(x) \leq r\right\}$. By the triangle inequality, $\forall x, y \in B\left(n,\left\lfloor\frac{d-1}{2}\right\rfloor\right), d(x, y) \leq$ $d-1$. Therefore $N_{q}(n, t) \geq\left|B\left(n,\left\lfloor\frac{d-1}{2}\right\rfloor\right)\right|$, and $A_{q}(n, d) \leq$ $\frac{q^{n}}{B\left(n,\left\lfloor\frac{d-1}{2}\right\rfloor\right)}$.
The number $N_{q}(n, t)$ is well studied in extremal combinatorics [3] [5], and a closed form for it is known. Thus, exact expressions of $N_{q}(n, t)$ can be used to derive better upper bounds on $A_{q}(n, d)$. For instance, if $n-t$ is even, $N_{2}(n, t)=\sum_{i=0}^{\frac{n-t}{2}}\left(\begin{array}{c}n \\ i\end{array}\right)$. Thus, in this case, $B\left(n,\left\lfloor\frac{d-1}{2}\right\rfloor\right)$ is a maximal anticode. However, when $n-t$ is odd, $N_{2}(n, t)=$ $2 \sum_{i=0}^{\frac{n-t-1}{2}}\left(\begin{array}{c}n-1 \\ i\end{array}\right)$ [3, Thm. Kl] and [8]. Therefore, we obtain the following lemma.

Lemma 5:

$$
A(n, d) \leq \frac{2^{n-1}}{\sum_{i=0}^{\frac{d-2}{2}}\left(\begin{array}{c}
n-1 \\
i
\end{array}\right)}, \quad \text { if } d \text { is even. }
$$

Notice that the above bound is tighter than the Hamming bound for even $d$ since

$$
2 \sum_{i=0}^{\frac{d-2}{2}}\left(\begin{array}{c}
n-1 \\
i
\end{array}\right)-\sum_{i=0}^{\frac{d-2}{2}}\left(\begin{array}{l}
n \\
i
\end{array}\right)=\left(\begin{array}{c}
n-1 \\
\frac{d-2}{2}
\end{array}\right)>0 .
$$

This new improved Hamming bound was recently proven in [9] using different techniques than the one presented here.

Next we give a new upper bound on $A_{q}(n, d)$ for alphabets of arbitrary size.

Lemma 6: For $q \geq 3, t=n-d+1$ and $r=$ $\left\lfloor\min \left\{\frac{n-t}{2}, \frac{t-1}{q-2}\right\}\right\rfloor$,

$$
A_{q}(n, d) \leq \frac{q^{t+2 r}}{\sum_{i=0}^{r}\left(\begin{array}{c}
t+2 r \\
i
\end{array}\right)(q-1)^{i}} .
$$

Proof: The proof follows from Corollary 1 and Thm. 2 in [5] or the Diametric Theorem of [3].

Note that for $q \geq t+1, N_{q}(n, t)=q^{n-t}$ [5, Corollary 1], i.e. a maximal anticode would be the trivial set $T(n, t)$ described in the proof of Lemma 3 . In this case, the bound of (3) boils down to the Singleton bound.

For $d$ even and $n$ not much larger than $t$, the next lemma provides an improvement on the Hamming bound for nonbinary alphabets.

Lemma 7: For $d$ odd and $n \leq t+1+\frac{\log t}{\log (q-1)}$

$$
A_{q}(n, d) \leq \frac{q^{n-1}}{\sum_{i=0}^{\frac{d-2}{2}}\left(\begin{array}{c}
n-1 \\
i
\end{array}\right)(q-1)^{i}}
$$

Proof: Under the conditions of this lemma, $N_{q}(n, t)=$ $q \sum_{i=0}^{\frac{d-2}{2}}\left(\begin{array}{c}n-1 \\ i\end{array}\right)(q-1)^{i}$ [3, Eq. 1.7]. The result then follows from Corollary 1 .

\section{Bounds For Constant Weight Codes}

Let $A(n, 2 \delta, w)$ be the maximum possible number of codewords in a binary code of length $n$, constant weight $w$ and minimum distance $2 \delta$ [2], [10].

Define the graph $K(n, 2 \delta, w)$ as the graph whose vertices are all the binary sequences of length $n$ and weight $w$ and where two vertices $u, v$ are adjacent iff $d(u, v) \geq 2 \delta$. It can be easily seen that $A(n, 2 \delta, w)=\omega(K(n, 2 \delta, w))$.

Let $\left(\begin{array}{c}{[n]} \\ w\end{array}\right)$ denote the set of all subsets of $[n]=$ $\{1,2, \ldots, n\}$ of order $w$. There is a natural bijection $\nu$ between $\mathrm{V}(K(n, 2 \delta, w))$ and $\left(\begin{array}{c}{[n]} \\ w\end{array}\right)$. Namely, $\forall u \in \mathrm{V}(K(n, 2 \delta, w))$, $\nu(u)=U=\{i ; u(i)=1\}$. 
Lemma 8: $\forall p, q \in \mathrm{V}(K(n, 2 \delta, w)), p \sim q$ iff $|P \cap Q| \leq$ $w-\delta$ where $P=\nu(q)$ and $Q=\nu(q)$.

Proof: $2 \delta \leq d(p, q)=|(P \cap \bar{Q}) \cup(\bar{P} \cap Q)|=2 w-$ $2|P \cap Q|$.

Lemma 9: $K(n, 2 \delta, w)$ is vertex transitive.

Proof: For any two vertices $p, q$ of $K$, any bijection on $[n]$ such that the image of $P=\nu(p)$ is $Q=\nu(q)$, takes $p$ to $q$ and belongs to $\operatorname{Aut}(K)$.

The first result that follows directly from Lemma 9 is the Bassalygo-Elias inequality [10]. We first recall some additional results in graph theory.

Definition 5 (Graph Homomorphism): Let $X$ and $Y$ be two graphs. A mapping $f$ from $\mathrm{V}(X)$ to $\mathrm{V}(Y)$ is a homomorphism if $\forall x, y \in \mathrm{V}(X) x \sim y \Rightarrow f(x) \sim f(y)$.

Theorem 2: If $Y$ is vertex transitive and there is a homomorphism from $X$ to $Y$, then

$$
\frac{|V(X)|}{\alpha(X)} \leq \frac{|V(Y)|}{\alpha(Y)}
$$

Proof: An application of Lemma 7.14.2 in [7].

Lemma 10 (Bassalygo-Elias inequality):

$$
A(n, d) \leq \frac{2^{n}}{\left(\begin{array}{c}
n \\
w
\end{array}\right)} A(n, d, w)
$$

Proof: Consider the two graphs $Y=\bar{H}(n, d)$ and $X=\bar{K}(n, d, w) . Y$ is vertex transitive. Since $X$ is an induced subgraph of $Y$, the inclusion map is a homomorphism that takes $X$ to $Y$. The result then follows from applying Thm. 2

By the same token, we can show the below equalities Lemma 11:

$$
\begin{aligned}
& A(n, d, w) \leq \frac{n-w+1}{w} A(n, d+2, w-1) \\
& A(n, d, w) \leq \frac{n+1}{w+1} A(n+1, d+2, w+1) \\
& A(n, d, w) \leq \frac{n}{w} A(n-1, d, w-1) \\
& A(n, d, w) \leq \frac{n}{n-w} A(n-1, d, w)
\end{aligned}
$$

Proof: We start by proving inequality [5. Let $\phi$ be a mapping from $\left(\begin{array}{c}{[n]} \\ w-1\end{array}\right)$ to $\left(\begin{array}{c}{[n]} \\ w\end{array}\right)$, such that $\forall P \in\left(\begin{array}{c}{[n]} \\ w-1\end{array}\right), P \subset$ $\phi(P) . \phi$ is a homomorphism from $K(n, d+2, w-1)$ to $K(n, d, w)$. In fact, $\forall P, Q \in K(n, d+2, w-1)$ such that $P \sim Q,|\phi(P) \cap \phi(Q)| \leq|P \cap Q|+2 \leq w-1-(d+2) / 2+2=$ $w-d / 2$ (by Lemma 8). Therefore, $\phi(P) \sim \phi(Q)$. The inequality then follows by applying Thm. 2

To prove inequality 6, take the homomorphism $\phi$ from $K(n+1, d+2, w+1)$ to $K(n, d, w)$ to be $\phi(X)=X \backslash$ $\left\{\max _{x \in X} x\right\}, \forall X \in\left(\begin{array}{c}{[n+1]} \\ w+1\end{array}\right)$.

The rest of the inequalities can be proved similarly by considering the corresponding graphs and taking the homomorphism to be the inclusion map.

The first two inequalities are new, whereas inequalities 7 and 8 were first proven by Johnson in [11].

Similarly, we can show the following inequalities regarding $A_{q}(n, d)$.
Lemma 12:

$$
\begin{aligned}
& A_{q}(n, d) \leq \frac{1}{q} A_{q}(n+1, d+1) \\
& A_{q}(n, d) \leq q A_{q}(n-1, d) \\
& A_{q}(n, d) \leq \frac{q^{n}}{(q-1)^{n}} A_{q-1}(n, d, w)
\end{aligned}
$$

Lemma 13: Let $t=w-\delta+1$.

$$
A(n, 2 \delta, w) \leq \frac{\left(\begin{array}{l}
n \\
w
\end{array}\right)}{\left(\begin{array}{l}
n-t \\
w-t
\end{array}\right)}
$$

Proof: Let $G=K(n, d, w)$. Since $G$ is vertex transitive, we have

$$
A(n, 2 \delta, w) \alpha(G) \leq|V(G)|=\left(\begin{array}{l}
n \\
w
\end{array}\right) .
$$

Define $M(n, w, s)$ as in [4] to be the maximum number of subsets of $[n]$ of order $w$ that intersect pairwise in at least $s$ elements. By Lemma $8, \alpha(G)=M(n, w, t)$. But, $M(n, w, t) \geq\left(\begin{array}{c}n-t \\ w-t\end{array}\right)$ (for instance, consider the system of all subsets of $[n]$ of order $w$ that contain the set $\{1,2, \ldots, t\})$.

The bound of Lemma 13 is actually the same as the one in Thm. 12 in [10] which was given with a different proof.

One can improve on the bound of Lemma 13 by using the exact value of $M(n, w, t)$ [4]. It is known that for $n \geq(w-$ $t+1)(t+1), M(n, w, t)=\left(\begin{array}{c}n-t \\ w-t\end{array}\right)$ [13], [14]. However, this is not the case for lower values of $n$.

Lemma 14: Let $t=w-\delta+1$ and $r=\max \left\{0,\left\lceil\frac{\delta(w-\delta)}{n-d}-\right.\right.$ $17\}$, then

$$
A(n, 2 \delta, w) \leq \frac{\left(\begin{array}{c}
n \\
w
\end{array}\right)}{\sum_{i=t+r}^{w}\left(\begin{array}{c}
t+2 r \\
i
\end{array}\right)\left(\begin{array}{c}
n-t-2 r \\
w-i
\end{array}\right)}
$$

with $\left(\begin{array}{l}n \\ k\end{array}\right)=0$ when $k>n$.

Proof: (sketch) $A(n, d, w) \leq \frac{\left(\begin{array}{l}n \\ w\end{array}\right)}{M(n, w, t)}$, then use the exact value of $M(n, w, t)$ given by the main theorem of [4].

\section{Bounds For Doubly Bounded Weight Codes}

Let $T\left(w_{1}, n_{1}, w_{2}, n_{2}, d\right)$ be the maximum number of codewords in a doubly constant weight binary code of minimum distance $d$, length $n=n_{1}+n_{2}$ and constant weight $w=$ $w_{1}+w_{2}$, where the first $n_{1}$ entries of each codewords have exactly $w_{1}$ ones [12]. $T^{\prime}\left(w_{1}, n_{1}, w_{2}, n_{2}, d\right)$ is defined similarly but where the first $n_{1}$ entries of each codewords have at most $w_{1}$ ones [10].

\section{Lemma 15:}

$$
\begin{aligned}
A(n, d, w) & \leq \frac{\left(\begin{array}{c}
n_{1}+n_{2} \\
w_{1}+w_{2}
\end{array}\right)}{\left(\begin{array}{c}
n_{1} \\
w_{1}
\end{array}\right)\left(\begin{array}{c}
n_{2} \\
w_{2}
\end{array}\right)} T\left(w_{1}, n_{1}, w_{2}, n_{2}, d\right) \\
A(n, d) & \leq \frac{2^{n}}{\sum_{i=0}^{w_{1}}\left(\begin{array}{c}
n_{1} \\
i
\end{array}\right)\left(\begin{array}{c}
n_{2} \\
w_{1}+w_{2}-i
\end{array}\right)} T^{\prime}\left(w_{1}, n_{1}, w_{2}, n_{2}, d\right)
\end{aligned}
$$

Proof: Same as Lemma 10.

Note that inequality (11) was first proven in [12], whereas inequality (12) is new. Several other bounds on $T\left(w_{1}, n_{1}, w_{2}, n_{2}, d\right)$ known in literature, such as Theorem 36 in [10], can be also easily obtained in the same way. The next lemma establishes some additional new bounds. 
Lemma 16:

$$
\begin{aligned}
& T\left(w_{1}, n_{1}, w_{2}, n_{2}, d\right) \leq\left(\begin{array}{c}
n_{2} \\
w_{2}
\end{array}\right) A\left(n_{1}, w_{1}, d-2 w_{2}\right) \text { if } d-2 w_{2} \geq 0 \\
& T\left(w_{1}, n_{1}, w_{2}, n_{2}, d\right) \leq\left(\begin{array}{c}
n_{1} \\
w_{1}
\end{array}\right) A\left(n_{2}, w_{2}, d-2 w_{1}\right) \text { if } d-2 w_{1} \geq 0 \\
& T\left(w_{1}, n_{1}, w_{2}, n_{2}, d\right) \leq \frac{n_{1}-w_{1}+1}{w_{1}} T\left(w_{1}-1, n_{1}, w_{2}, n_{2}, d+2\right) \\
& T\left(w_{1}, n_{1}, w_{2}, n_{2}, d\right) \leq \frac{n_{1}+1}{w_{1}+1} T\left(w_{1}+1, n_{1}+1, w_{2}, n_{2}, d+2\right) \\
& T\left(w_{1}, n_{1}, w_{2}, n_{2}, d\right) \leq \frac{n_{2}-w_{2}+1}{w_{2}} T\left(w_{1}, n_{1}, w_{2}-1, n_{2}, d+2\right) \\
& T\left(w_{1}, n_{1}, w_{2}, n_{2}, d\right) \leq \frac{n_{2}+1}{w_{2}+1} T\left(w_{1}, n_{1}, w_{2}+1, n_{2}+1, d+2\right)
\end{aligned}
$$

\section{OTHER ApPlications}

In this section we demonstrate how the above techniques can be helpful in solving other problems. For instance, we show how to compute $N_{q}(n, 1)$, the maximum number of $q$ ary sequences of length $n$ intersecting pairwise in at least one position [3].

Lemma 17: $N_{q}(n, 1)=q^{n-1}$

Proof: Let $G=H_{q}(n, n) ; N_{q}(n, 1)=\alpha(G)$. Now, consider the set of $q$ sequences where the entries in the $i$-th sequence are all the same and equal to $i$, hence $\omega(G) \geq q$. But $\omega(G) \leq q$ since the first entries of all sequences in a clique in $G$ should contain different letters. Therefore, $\omega(G)=q$. By Lemma 2, we get $N_{q}(n, 1) \leq q^{n-1}$. But $N_{q}(n, 1) \geq q^{n-1}$ (see the proof of Lemma 3.

The next lemma gives the chromatic number of certain Hamming graphs.

Lemma 18: $\chi\left(H_{q}(n, d)\right)=q^{n-d+1}$, for $q \geq n-d+2$, $1 \leq d \leq n$.

Proof: From the definitions, it follows that for any graph $G, \chi(G) \geq \frac{|\mathrm{V}(G)|}{\alpha(G)}$. But, $\alpha\left(H_{q}(n, d)\right)=q^{d-1}[5$, Corollary 1]. Therefore, $\chi\left(H_{q}(n, d)\right) \geq \frac{q^{n}}{q^{d-1}}=q^{n-d+1}$.

Let $\phi$ be a mapping from $\Sigma^{n}$ to $\Sigma^{n-d+1}$ consisting of deleting the last $d-1$ entries of a sequence. $\phi$ is a homomorphism from $H_{q}(n, d)$ to $H_{q}(n-d+1,1)=K^{n-d+1}$, where $K^{\ell}$ is the complete graph on $\ell$ vertices. Therefore, $\chi\left(H_{q}(n-d+1,1)\right) \leq \chi\left(K^{n-d+1}\right)=q^{n-d+1}[7$, Lemma 1.4.1].

Let $v(G)$ be the Lovász upper bound [15] on the zero error capacity $\Theta(G)$ [16] of a graph $G$. We recall the following two results of [15].

Lemma 19: $\alpha(G) \leq \Theta(G) \leq v(G)$

Theorem 3: If $G(V, E)$ is vertex transitive then $v(G) v(\bar{G})=|V|$.

In the following, we give a partial answer to a question raised in the conclusion of [15], namely "Find further graphs with $v(G)=\Theta(G)$ ".

Lemma 20: The following graphs satisfy $v(G)=\Theta(G)$

1) $H_{q}(n, d)$ when there exists a q-ary perfect code of length $n$ and minimum distance $d$.

2) $H_{q}(n, d)$ when $q \geq n-d+2$ and there exists a q-ary MDS code of length $n$ and minimum distance $d$.

3) $H_{q}(n, n)$.
Proof: Let $G$ be a vertex transitive graph such that $\alpha(G) \alpha(\bar{G})=|\mathrm{V}(G)|$. Then, applying Lemma 19 to $G$ and $\bar{G}$ and multiplying the two resulting equations we get $\frac{\Theta(G)}{v(G)}=$ $\frac{v(\bar{G})}{\Theta(G)} \geq 1$. Therefore, $\Theta(G)=v(G)$. One can check that the graphs $G$ belonging to the three families mentioned above satisfy $\alpha(G) \alpha(\bar{G})=|\mathrm{V}(G)|$.

\section{CONCLUSION}

We constructed vertex transitive graphs where a code corresponds to a clique and an anti-code to an independent set. Thus, we established a connection between the maximal order of codes and that of anti-codes. Using intersection theorems for systems of finite sets and that of finite sequences, we provided a framework where several known bounds on code size follow easily and new inequalities can be derived.

Several questions naturally arise here.

1) What are the zero error capacities of the graphs $H$ and $K$ and their complements $\bar{H}$ and $\bar{K}$ ? What are the values of the $v$ function of these graphs. Note, that these quantities can be useful to derive bounds for $A_{q}(n, d)$ and $A(n, d, w)$ using Lemma 19 and Thm. 3 .

2) From a graph theoretical standpoint, trying to extend the result of Lemma 18 by finding the chromatic number of the above graphs is also an interesting question, and can have applications to coding theory and cryptography.

3) Perfect codes are codes who achieve the Hamming bound. We gave here many upper bounds lower than the Hamming bound in specific cases (Lemma 5, (3), Lemma 7 and (10); thus ruling out the existence of perfect codes there. It is an interesting question to find whether there exist "nearly perfect codes" that can achieve these new bounds.

\section{REFERENCES}

[1] F. J. Macwilliams and N. J. A. Sloane, The Theory of Error-CorrectingCodes, North-Holland, 1977.

[2] N. J. A. Sloane, "Unsolved Problems in Graph Theory Arising from the Study of Codes," Graph Theory Notes of New York, 1989.

[3] R. Ahlswede and L. H. Khachatrian, "The Diametric Theorem in Hamming Spaces - Optimal anticodes," Adv. in Appl. Math, vol. 20, pp. 429-449, 1998.

[4] R. Ahlswede and L. H. Khachatrian, " The Complete Intersection Theorem for Systems of Finite Sets," European Journal of Combinatorics, vol. 18, pp. 125-136, 1997

[5] P. Frankl and N. Tokushige, "The Erdős-Ko-Rado Theorem for Integer Sequences," Combinatorica, vol. 19, pp. 55-63, 1999.

[6] R. Diestel, Graph Theory, Springer, 2006.

[7] C. Godsil and G. Royle , Algebraic Graph Theory, Springer, 2001.

[8] D. J. Kleitman, "On a Combinatorial Conjecture of Erdôs," J. of Combinatorial Theory, vol. 1, pp. 209-214, 1966.

[9] R. Matsumoto, K. Kurosawa, T. Itoh, T. Konno and T. Uyematsu, "Primal-Dual Distance Bounds of Linear Codes with Applications to Cryptography," IEEE Trans. Inform. Th., vol. 52, pp. 4251-4256, 2006.

[10] E. Agrell, A. Vardy and K. Zeger, " Upper Bounds for Constant-Weight Codes," IEEE Trans. Inform. Theory, vol. 46, pp. 2373-2395, 2000.

[11] S. M. Johnson, "A new upper bound for error-correcting codes," IRE Trans. Inform. Theory, vol. IT-8, pp. 203-207, 1962.

[12] V. I. Levenshtein, "Upper Bound Estimates For Fixed-Weight Codes," Probl. Inform. Trans., vol. 7, pp. 281-287, 1974.

[13] P. Erdős, C. Ko and R. Rado, " Intersection Theorems for systems of finite sets," Quart. J. Math. Oxford, vol. 12, pp. 313-320, 1961.

[14] R. M. Wilson, "The exact bound on the Erdos-ko-Rado Theorem," Combinatorica, vol. 4, pp. 247-260, 1984.

[15] L. Lovász, "On the Shannon Capacity of a Graph," IEEE Trans. Inform. Theory, vol. IT-25, pp. 1-7, 1979.

[16] C.E. Shannon, "The Zero Error Capacity of a Noisy Channel," vol. IT-2, pp. 8-19, 1956. 